\title{
Quantum confinement and band offsets in amorphous silicon quantum wells
}

\author{
K. Jarolimek, ${ }^{1}$ R. A. de Groot, ${ }^{2}$ G. A. de Wijs, ${ }^{2}$ and M. Zeman ${ }^{1}$ \\ ${ }^{1}$ PVMD/DIMES, Delft University of Technology, Feldmannweg 17, 2600 GB Delft, The Netherlands \\ ${ }^{2}$ Radboud University Nijmegen, Institute for Molecules and Materials, Heyendaalseweg 135, 6525 AJ Nijmegen, The Netherlands
}

(Received 9 September 2013; revised manuscript received 10 August 2014; published 18 September 2014)

\begin{abstract}
Quantum wells (QWs) are nanostructures consisting of alternating layers of a low and high band-gap semiconductor. The band gap of QWs can be tuned by changing the thickness of the low band-gap layer, due to quantum confinement effects. Although this principle is well established for crystalline materials, there is still controversy for QWs fabricated from amorphous materials: How strong are the confinement effects in amorphous QWs, where, because of the disorder, the carriers are localized to start with? We prepare an atomistic model of QWs based on a-Si:H to gain insight into this problem. The electronic structure of our atomistic QWs model is described with first-principles density functional theory, allowing us to study the confinement effects directly. We find that the quantum confinement effect is rather weak, compared to experimental results on a similar system.
\end{abstract}

DOI: 10.1103/PhysRevB.90.125430

PACS number(s): 73.21.Fg, 71.23.Cq, 71.15.Mb, 71.15.Pd

\section{INTRODUCTION}

Quantum wells (QWs) consist of alternating layers of two semiconductors with different band gaps. The layers with low and high band gaps are referred to as "well" and "barrier," respectively. The thickness of the well layer is in the nanometer range, resulting in the quantum confinement of charge carriers. By changing the thickness of the well one can engineer the band gap of the metamaterial.

Nowadays QWs are implemented in devices such as lasers and detectors [1]. A considerable part of the experience with QWs structures comes from crystalline semiconductors (mostly group III-V). The two materials have to be lattice matched in order to grow epitaxially on top of each other. In order to remove this restriction, Abeles and Tiedje fabricated QWs from amorphous semiconductors [2]. Another advantage is that amorphous semiconductors can be deposited relatively cheaply over large areas [3]. This is important for optoelectronic devices, such as light-emitting diodes and solar cells.

Soon after the fabrication of the first amorphous QWs reports on the increase of the optical gap [2,4-8], resonant tunneling [5,9], and the shift of the photoluminescence peak followed $[10,11]$. The observed effects were attributed to quantum confinement of charge carriers, often employing the quantum well model to fit the data. The existence and magnitude of quantum effects in amorphous/amorphous nanostructures is, however, still debated. Collins and Huang claim that the use of the Tauc law can introduce systematic errors in optical gaps when used on multilayers [12]. Beaudoin et al. observe the usual confinement effects for the Tauc gap, but not for the Cody gap [13]. Steps in the current-voltage characteristics were attributed to other effects than resonant tunneling $[14,15]$. Reviews on the subject can be found in Bernhard et al. [16] and Koehler [17].

In order to gain new insight into this problem, we have prepared an atomistic model of a hydrogenated amorphous silicon (a-Si:H) based multilayer. a-Si:H is a prototype amorphous semiconductor that forms nonstoichiometric compounds with carbon or nitrogen. These compounds have relatively high band gaps and are used as the barrier layer. Throughout this study we select silicon nitride $(\mathrm{a}-\mathrm{SiN}: \mathrm{H})$ as the barrier. The atomistic model allows us to study quantum confinement effects directly without the need for transport or optical measurements. The interpretation of such measurements could be complicated by interface effects or the inappropriate use of models to extract the optical band gap.

The investigation of amorphous QWs or amorphous/amorphous interfaces with atomistic models has been limited so far, mostly due to the computational cost. Kuzuu et al. [18] investigated the structure of the $\mathrm{a}-\mathrm{SiO}_{2} / \mathrm{a}-\mathrm{SiO}_{2}$ interface. Several studies have focused on interfaces of crystalline silicon with either pure amorphous silicon [19-27] or a- $\mathrm{SiO}_{2}$ glass [28-34]. Only a part of these studies addresses also the band offsets between the materials [24,27-29,31,33,34]. Calculations on the $\mathrm{c}-\mathrm{Si}$ and hydrogenated amorphous silicon were performed by Tosolini et al. [35] and Van de Walle et al. [27]. The preparation of structural models of the amorphous materials usually relies on interatomic model potentials with the exception of Pasquarello et al. [32,34], who used first-principles molecular dynamics (MD) [36]. This level of modeling is necessary to describe the various structural and chemical environments that can occur at the interface and is also used throughout this work.

The paper is organized as follows. Section II provides technical details on the calculations. The preparation of the structural model of the QWs is described in Sec. III. In Sec. IV we present a method to determine band edges and band gaps in amorphous semiconductors. The magnitude of the quantum confinement effect for different systems is studied in Sec. V. Results on the band offsets in the QWs are given in Sec. VI. Finally conclusions are presented in Sec. VII.

\section{TECHNICAL DETAILS}

All calculations were performed on the level of density functional theory (DFT) with the Vienna ab initio simulation package (VASP) [37,38]. Electron-ion interactions were described using the projector augmented wave method $[39,40]$.

Molecular dynamics calculations were performed with a 1 fs time step. During the whole MD run and the relaxation, we use only the $\Gamma$ point for Brillouin zone sampling. A "soft" nitrogen potential, supplied with VASP, with a $250 \mathrm{eV}$ kinetic 
TABLE I. Band gaps of crystalline $\mathrm{Si}$ and $\alpha-\mathrm{Si}_{3} \mathrm{~N}_{4}$ calculated with different levels of accuracy. All values are in electron volts.

\begin{tabular}{llcc}
\hline \hline & $\mathrm{Si} \Gamma \rightarrow \Gamma$ & $\mathrm{Si} \Gamma \rightarrow \mathrm{X}$ & $\beta-\mathrm{Si}_{3} \mathrm{~N}_{4} \Gamma \rightarrow \Gamma$ \\
\hline GGA-PBE & 2.57 & 0.72 & 4.55 \\
HSE06 & 3.35 & 1.32 & 6.03 \\
$G_{0} W_{0}$ & 3.19 & 1.19 & 6.29 \\
Experiment & $3.05^{\mathrm{a}}$ & $1.25^{\mathrm{a}}$ & - \\
& $3.34-3.36^{\mathrm{b}}$ & & \\
\hline \hline
\end{tabular}

${ }^{\mathrm{a}}$ Reference [47].

${ }^{\mathrm{b}}$ Reference [48]

energy cutoff was used. The potential uses core radii of 0.794 and $1.005 \AA$ for the $s$ and $p$-partial waves, respectively. Two channels per angular momentum quantum number were used. The performance of the potentials was tested on the $\alpha$ and $\beta$ phases of $\mathrm{Si}_{3} \mathrm{~N}_{4}$. The calculated cell parameters were higher than experimental ones by less than $1 \%$. The difference in cell parameters between the "soft" and "normal" potential was $\sim 0.1 \%$. The "normal" potential employs smaller core radii of 0.635 and $0.794 \AA$ for the $s$ and $p$-partial waves, respectively. Also this time two channels per angular momentum quantum number were used. The above described tests, as well as all dynamic calculations were performed with the generalized gradient approximation (GGA) using a PW91 functional [41].

Although the GGA gives accurate structural properties it is known to underestimate band gaps. In order to obtain realistic band gaps and offsets, all static calculations are performed with a hybrid functional. This type of functional includes a part of exact exchange from Hartree-Fock theory. Tests on 40 semiconductors show a dramatic improvement in band-gap values [42]. We used the HSE06 hybrid functional $[43,44]$ with a screening parameter of $0.2 \AA^{-1}$. A Perdew-Burke-Ernzerhof (PBE) nitrogen potential with a $400 \mathrm{eV}$ cutoff was used. The Brillouin zone is sampled at a single point $(1 / 4,1 / 4,1 / 4)$, as suggested by Baldereschi [45]. Density of states is calculated with a Gaussian smearing with a width of $0.05 \mathrm{eV}$.

For calculations on c-Si QWs in vacuum the cutoff was reduced to $250 \mathrm{eV}$. The Brillouin zone sampling is adjusted depending on the dimension of the supercell. The conventional c-Si cell (cube of $5.43 \AA$ ) was sampled with a $6 \times 6 \times$ $6 / 3 \times 3 \times 3$ mesh. The largest cell, which is $5 \times 5.43 \AA$ in the direction perpendicular to the slab, was sampled with a $6 \times 1 \times 6 / 3 \times 1 \times 3$ mesh. In the HSE06 calculations the second mesh is reduced by a factor of 2 and is used to evaluate the Fock exchange potential [46]. For relaxation the first mesh was used.

The performance of the hybrid functional was tested on crystals of silicon and $\alpha-\mathrm{Si}_{3} \mathrm{~N}_{4}$. The GGA-PBE functional clearly underestimates the band gaps of both semiconductors (see Table I). The hybrid functional yields higher band gaps and is in good agreement with $G_{0} W_{0}$ calculations and experiments, where available. The $G_{0} W_{0}$ method is successful in describing the excited-state properties of solids, although at an increased computational cost [49]. Tests on a wide range of solids give band gaps with a mean absolute relative error of 10\% [50]. During calculations on c-Si the kinetic energy cutoff was set to $250 \mathrm{eV}$. The cutoff was increased in the case of $\beta-\mathrm{Si}_{3} \mathrm{~N}_{4}$ to $400 \mathrm{eV}$ (GGA-PBE and HSE06) and to $420 \mathrm{eV}\left(G_{0} W_{0}\right)$.
Partial occupancies of states were determined with a Gaussian smearing with a width of $0.05 \mathrm{eV}$. All calculations used a $k$-point mesh centered at $\Gamma$. The Brillouin zone of the $\mathrm{c}-\mathrm{Si}$ cell was sampled as follows: $12 \times 12 \times 12$ (GGA-PBE), $12 \times$ $12 \times 12 / 6 \times 6 \times 6$ (HSE06) and $6 \times 6 \times 6\left(G_{0} W_{0}\right)$. For the $\beta$-Si ${ }_{3} \mathrm{~N}_{4}$ cell we used the following $k$-point mesh: $6 \times 6 \times 16$ (GGA-PBE), $6 \times 6 \times 16 / 3 \times 3 \times 8$ (HSE06), and $3 \times 3 \times 8$ $\left(G_{0} W_{0}\right)$. The single-shot $G_{0} W_{0}$ used a GGA-PBE calculation as a starting point.

\section{PREPARATION OF THE STRUCTURE}

A model of the QWs was constructed by merging cells of a-Si:H and a-SiN:H. The cells were prepared previously with the "cooling from liquid" method [51,52]. This is a common method that simulates rapid cooling of a melt, a process similar to the formation of glasses. The densities of the silicon and silicon nitride cells were taken from experiments and were 2.0 and $3.0 \mathrm{~g} / \mathrm{cm}^{3}$, respectively [53]. The final cell has composition $\mathrm{Si}_{410} \mathrm{~N}_{194} \mathrm{H}_{112}$ and approximate dimensions $16 \times 32 \times 16 \AA^{3}$. The dimensions of the individual cells were changed to minimize their combined total energy. First both cells were expanded isotropically from 16.593 to $16.972 \AA$ (by $2.3 \%$ ). Next the silicon cell was compressed by $0.49 \AA$ $(2.9 \%)$ in the $y$ direction. Conversely, the nitride cell was expanded (also along $y$ ) by the same amount. Finally, we shift the cells relative to each other along the interface plane (defined by $x$ and $z$ vectors) in order to prevent atoms from being too close in the newly formed interface. We will denote this cell as the initial model. Note, that for all calculations periodic boundary conditions are used. This means that the cell (containing a silicon and silicon nitride part) is repeated in the $x, y$, and $z$ directions. The resulting structure thus consists of an infinite number of identical quantum wells with two different interfaces.

In order to test the influence of the structure on the electronic properties (quantum confinement, band offsets) we generate three more models. The relaxed model is obtained by a relaxation of the initial cell, during which atoms move to the nearest potential energy minimum. In order to let atoms rearrange into a more energetically favorable structure, we heat the cell to $1818 \mathrm{~K}$. The annealing cycle lasts 4 ps and leads to a complete melting of the silicon. The silicon nitride network is unaltered, since it has a much higher melting temperature. Next the cell is cooled back to $300 \mathrm{~K}$. We utilize two different cooling rates, 1.380 or $0.023 \mathrm{~K} / \mathrm{fs}$, that lead to the fast and slow models. The number of coordination defects and cohesive energies for different models is summarized in Table II. One can see that the slow anneal is quite effective at removing coordination defects, resulting in a concentration of $\sim 3$ at. $\%$. We note that annealing at elevated temperatures promotes hydrogen to a mobile state. A part of the hydrogen becomes trapped in the interface region, where it passivates defects. The difference between the cohesive energies of the different interface models with respect to the combined cohesive energy of the isolated cells, gives an estimate of the interface energy. The initial, relaxed, and fast models all have a positive interface energy while the slow model has a negative interface energy of $-0.35 \mathrm{~J} / \mathrm{m}^{2}$ [54]. We conclude that the slow cell is superior 
TABLE II. Summary of coordination defects present in different models. The following cutoff distances were used: $r_{\mathrm{Si}-\mathrm{Si}}=2.65 \AA$, $r_{\mathrm{Si}-\mathrm{N}}=2.00 \AA, r_{\mathrm{Si}-\mathrm{H}}=1.65 \AA, r_{\mathrm{N}-\mathrm{H}}=1.15 \AA$, and $r_{\mathrm{H}-\mathrm{H}}=0.85 \AA$. $\mathrm{Si} 2$ denotes a twofold coordinated silicon atom. Artificial Si-Si bonds inside "square structures" are discarded from the count (see Ref. [52] for details). The column denoted by $D_{\text {tot }}$ contains the total number of structural defects. The last column contains cohesive energies of cells per atom in $\mathrm{eV}$. In the first row we give the sum of defects as well as cohesive energies of the isolated cells.

\begin{tabular}{lcccccccccccc}
\hline \hline Cell & $\mathrm{Si} 2$ & $\mathrm{Si} 3$ & $\mathrm{Si} 5$ & $\mathrm{Si} 6$ & $\mathrm{H} 0$ & $\mathrm{H} 2$ & $\mathrm{~N} 1$ & $\mathrm{~N} 2$ & $\mathrm{~N} 4$ & $D_{\text {tot }}$ & $E(\mathrm{eV})$ \\
\hline Isolated & 0 & 16 & 8 & 0 & 2 & 0 & 0 & 2 & 2 & 30 & -6.188 \\
Initial & 3 & 40 & 21 & 2 & 5 & 1 & 4 & 14 & 1 & 91 & -5.980 \\
Relaxed & 0 & 23 & 12 & 0 & 0 & 0 & 0 & 5 & 6 & 46 & -6.139 \\
Fast & 1 & 13 & 13 & 0 & 0 & 0 & 0 & 4 & 8 & 39 & -6.157 \\
Slow & 0 & 8 & 5 & 0 & 1 & 0 & 0 & 2 & 4 & 20 & -6.205 \\
\hline
\end{tabular}

to the other models and thus will be used in the study of the electronic structure.

\section{DETERMINATION OF THE BAND GAP}

The electronic structure of amorphous semiconductors is more complicated compared to crystalline ones. It consists of extended, tail, and defect states. Extended states are delocalized and similar to the states in a crystal. Disorder leads to the formation of localized states (tail states) at the band edges [55]. Tail states are separated from extended states by the mobility edge. The concentration of defects in amorphous semiconductors is relatively high and this results in a continuum of states within the band gap. The existence of band tails makes the definition of the band gap ambiguous. Over the years a number of definitions were devised [56]. In the following we will use the definition by Tauc, which is the simplest one. In this model the valence and conduction band density of states (DOS; $N_{v}$ and $N_{c}$ ) follow a square root dependence on energy:

$$
N_{v}(E)=\left\{\begin{array}{lll}
0 & \text { if } & E>E_{v} \\
N_{v 0} \sqrt{E_{v}-E} & \text { if } & E \leqslant E_{v}
\end{array}\right.
$$

and

$$
N_{c}(E)=\left\{\begin{array}{lll}
N_{c 0} \sqrt{E-E_{c}} & \text { if } & E \geqslant E_{c} \\
0 & \text { if } & E<E_{c},
\end{array}\right.
$$

where $N_{v 0}$ and $N_{c 0}$ are the valence and conduction band DOS prefactors. $E_{v}$ and $E_{c}$ are the valence and conduction band edges. Figure 1 illustrates the use of the Tauc model to determine the band gap of a-Si:H. It is clear that the model is valid only for the middle range of DOS values. We choose an interval that spans from $30 \%$ to $80 \%$ of the maximum DOS value (at $28 \times 10^{21} \mathrm{~cm}^{-3} \mathrm{eV}^{-1}$ ). This allows us to find the energy ranges to fit the Tauc model to the calculated DOS. Using a lower limit of $30 \%$ effectively means that we will rely on the extended states to define the position of the band edge and that we suppress the effect of tail and defect states. After performing a least square fit we obtain a band gap of $1.7 \mathrm{eV}$, which is quite close to the experimental Tauc gap of $1.8 \mathrm{eV}$ [57]. The standard error of the fit parameters was estimated from the variance-covariance matrix. On average the standard

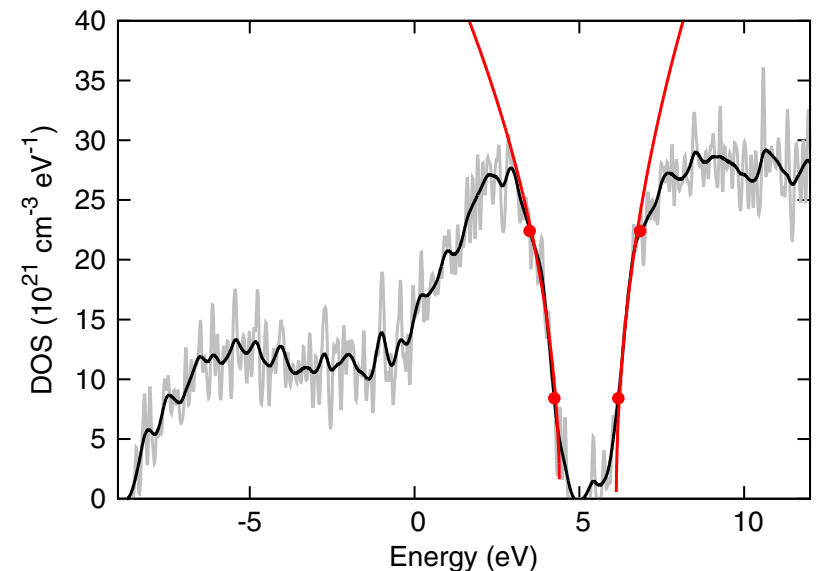

FIG. 1. (Color online) DOS of bulk a-Si:H (gray line) calculated with the HSE06 functional. The DOS is smoothened with a spline (black line). The valence and conduction band edges are fitted with a square root function (red lines). The range of data used for fitting is indicated with red points.

error in energy is $0.04 \mathrm{eV}$. Note that the DOS in Fig. 1 and in all subsequent figures is evaluated only in an 8 - $\AA$-wide slab. The slab is positioned in the middle of the cells that represent bulk silicon or silicon nitride. In the QWs model the slab is centered either in the silicon or silicon nitride part.

\section{QUANTUM CONFINEMENT}

Let us first investigate quantum confinement effects in a$\mathrm{Si}: \mathrm{H}$ surrounded by vacuum. We utilize the initial QWs model and replace the nitride part with vacuum, thus creating a $16 \AA$ silicon slab. Removing $4 \AA$ of silicon on both sides will result in an even thinner slab ( $8 \AA$ wide). Next the surfaces of both slabs are saturated with $\mathrm{H}$ atoms. Thanks to the way these cells were constructed, there exists a region ( $8 \AA$ wide) in all three cells (bulk, $16 \AA$ slab, $8 \AA$ slab) that has an identical atomic structure. As explained above we will use this region to calculate the DOS (see Fig. 2). The curves are aligned on

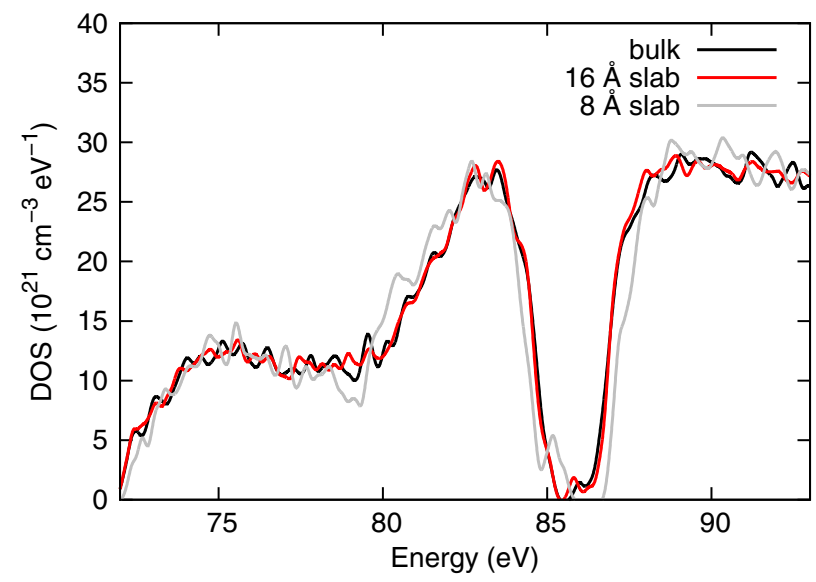

FIG. 2. (Color online) DOS of a-Si:H slabs in vacuum compared to bulk. All curves were smoothened with a spline and aligned at the core potential of a silicon atom. 


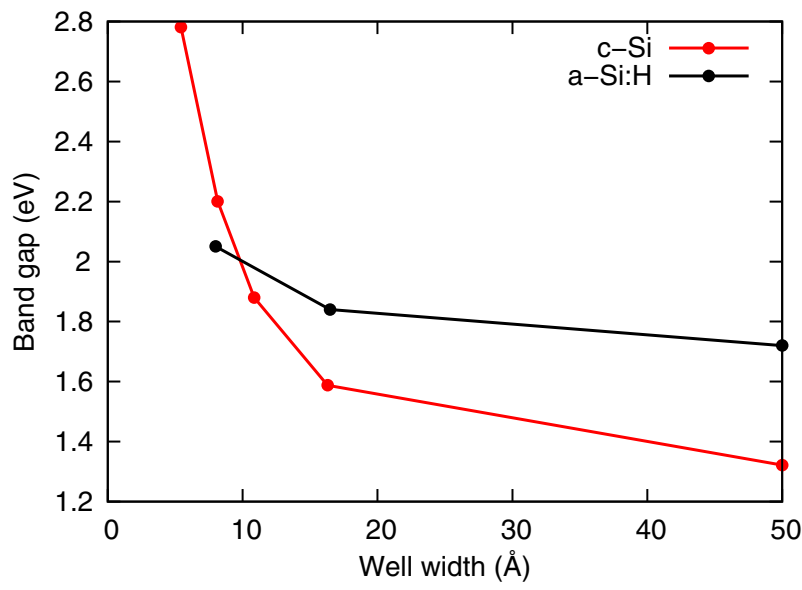

FIG. 3. (Color online) Band gaps of c-Si and a-Si:H slabs in vacuum as a function of slab thickness. Data points at $50 \AA$ represent bulk values.

the core potential of a silicon atom located approximately in the middle of the three silicon slabs. The surroundings of this atom are the same throughout all cells. We observe that the gap increases with a decreasing slab thickness. For the 16 and $8 \AA$ slabs the gaps are 1.8 and $2.1 \mathrm{eV}$, respectively. We also notice that the opening of the gap is symmetrical.

It is instructive to compare the magnitude of the confinement effect in a-Si:H to the crystalline case. We construct slabs of c-Si with thicknesses from one to three times the conventional unit cell ( $5.43 \AA$ ). In the lateral direction the slabs consist of only one unit cell. The (100) surfaces of the slabs are bulk terminated and saturated with hydrogen atoms. Both $\mathrm{Si}$ and $\mathrm{H}$ atoms were allowed to move during relaxation. Each supercell also contains two cells of vacuum. This amount of vacuum was found to be sufficient to separate the neighboring slabs [58].

Figure 3 shows a comparison of quantum confinement effects between amorphous and crystalline silicon. We find that the confinement effect in a-Si:H is rather weak. The bulk band gap of a-Si:H is larger than that of c-Si but for the $\sim 8 \AA$ slab the band gaps are comparable. We suggest that the different behavior of a-Si:H is due to localized states near the band edges. These states are already confined when in bulk. Thus a fabrication of a nanostructure made of a-Si:H that should lead to additional electron confinement will have a small effect. In Fig. 4 we plot the charge density of four states that correspond to the points in Fig. 1. The points define intervals that were used for the fitting of the Tauc gap. The top panel of Fig. 4 shows charge density that is averaged in the $x$ and $z$ directions (parallel to the interface). All of the states show some degree of localization and look qualitatively different from states in c-Si. The localization is the strongest for the state with a maximum near $11 \AA$ along the $y$ axis. When we sum all the charge that is contained in the 8 - $\AA$-wide region (indicated by the gray area) we obtain $57 \%$ of its total charge. For other states the percentages are within the $40 \%-60 \%$ range. The averaging procedure will, however, smooth the extremes of the three-dimensional function. In the bottom panel of Fig. 4 we plot the charge density along lines parallel to the $y$ axis.

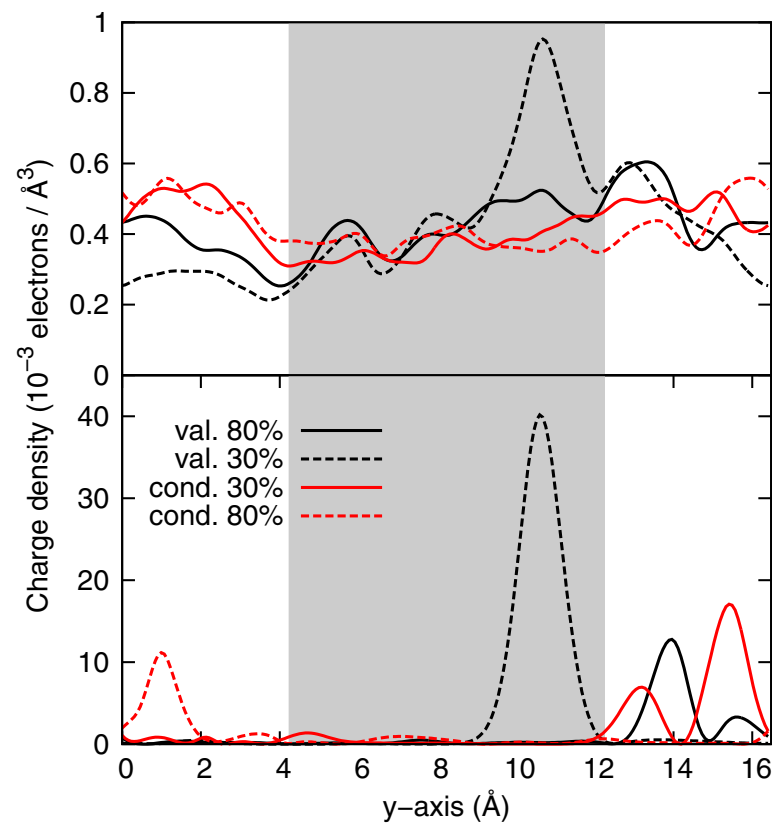

FIG. 4. (Color online) Charge density of four band edge states in bulk a-Si:H. In the top panel the charge density is averaged in the $x$ and $z$ direction. The bottom panel shows cuts through the 3 dimensional charge density along lines (parallel to the $y$-axis) that pass through the maximum value.

The lines pass through points of maximum charge density. This view shows a much stronger localization behavior.

Next we investigate quantum confinement in multilayers, where the well depth is smaller. For electrons (holes) it is determined by the conduction (valence) band offset at the interface. Figure 5 shows the DOS of a-Si:H in the QWs model. The band gap of the $16 \AA$ slab surrounded by silicon nitride is $1.5 \mathrm{eV}$. Similarly as in the case when the slab is surrounded by vacuum we do not find any quantum confinement effect. The band gap is even slightly reduced from the bulk a-Si:H value of $1.7 \mathrm{eV}$. The reduction is probably due to structural changes during the annealing cycle. We find that silicon atoms diffuse

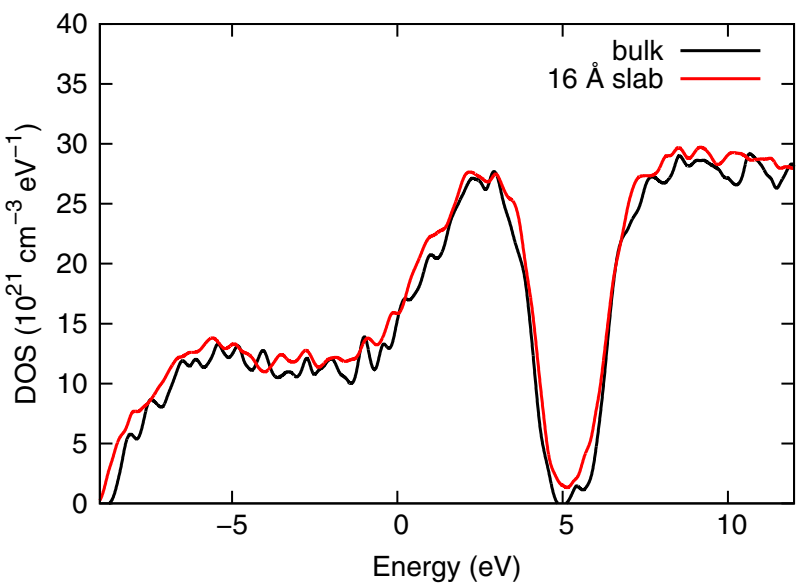

FIG. 5. (Color online) DOS of a-Si:H in the QW model compared to bulk. Both curves are smoothened with a spline. The alignment along the energy axis is arbitrary. 
from the edges of the a-Si:H cell into its middle part. In this part the density increases from $2.1 \mathrm{~g} / \mathrm{cm}^{3}$ (initial and relaxed models) to $2.4 \mathrm{~g} / \mathrm{cm}^{3}$ (fast and slow models). This will also cause an increase in the density of states and a reduction of the band gap. Note that the band gap in the relaxed model is the same as in the bulk.

Measurements on QWs with an identical silicon nitride composition as our models yield a Tauc gap of $2.3 \mathrm{eV}$ (for $13 \AA$ well thickness) [57]. The larger band gap can probably be explained also in purely optical terms: Since the barrier width is kept constant, decreasing the well width results in QWs with a higher average nitrogen concentration.

\section{BAND OFFSETS}

First-principles methods have been successfully applied to calculate band offsets between two crystalline materials $[59,60]$. A model of a crystalline interface is constructed by merging several conventional unit cells of material $A$ followed by cells of material $B$. This supercell is then used to extract the offset of the electrostatic potential between the two materials. The atomic-scale oscillation of the electrostatic potential is conveniently filtered out by averaging in a slab with a geometry of the conventional unit cell. When sufficiently far away from the interface, the averaged potential attains its bulk value. The band offsets are finally obtained as rigid shifts of the electronic structure of materials $A$ and $B$ by the potential offset. Here the term "electronic structure" denotes the position of the valence and conduction band edges in the bulk material.

Although this method works well for crystalline materials it is problematic to apply to amorphous solids. Disorder in the material makes it difficult to calculate a reliable potential average. Here the averaging will not filter out the oscillations in the potential completely. This is because our silicon and nitride cells are not composed of several identical unit cells. Naturally using a larger averaging slab will damp the oscillations. For a slab $8 \AA$ wide the standard deviation of the potential becomes $0.1 \mathrm{eV}$ for both materials. Another difficulty is that one cannot treat the bulk electronic structure as rigid because of the annealing that can alter the structure.

Here we follow a more straightforward approach for calculating band offsets that makes use of the local density of states. The DOS is calculated in thin slabs $(0.1 \AA)$ parallel to the interface. For a given eigenvalue the amount of states is proportional to the charge inside the slab. This method also circumvents the necessity to determine the averages of the electrostatic potential. In Fig. 6 the site-projected DOS of the QWs model is shown. We observe a symmetrical opening of the band gap when going from bulk silicon to the bulk silicon nitride regions. The valence and conduction band edges converge to their bulk values approximately $3 \AA$ from the interface border. Fluctuations in the band edges (especially in the conduction band) of silicon nitride are present. They originate from the inhomogeneities that are intrinsic to the silicon nitride with this particular composition and density [52].

To get a more quantitative view of the valence band offset we plot the DOS in an 8 - $\AA$-wide slab positioned in the center of the silicon and nitride cells making up the QWs model (see Fig. 7). In this way we obtain a valence and a conduction

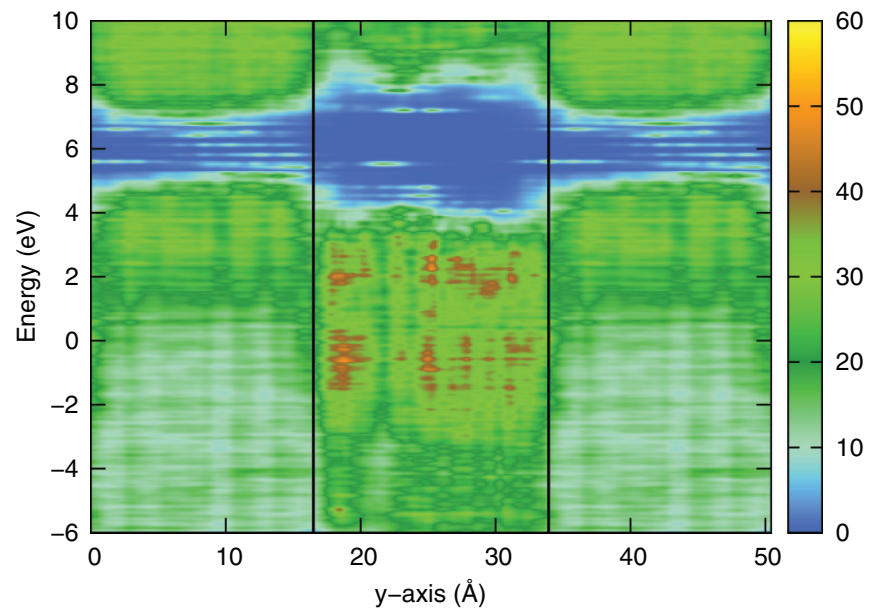

FIG. 6. (Color online) Local DOS (in $10^{21} \mathrm{~cm}^{-3} \mathrm{eV}^{-1}$ ) along the $y$ axis. The silicon cell is repeated in order to see both interfaces more clearly. Interfaces are marked by vertical lines. The band gap is centered around $6 \mathrm{eV}$.

band offset of 0.9 and $0.7 \mathrm{eV}$, respectively. The complete band diagram of the slow QWs model is drawn in Fig. 8. The band gaps of amorphous silicon and silicon nitride in the QWs model stay reasonably constant when compared with the isolated cells. In both cases they are reduced which can be explained by the change in structure during the annealing cycle.

\section{CONCLUSIONS}

Using DFT molecular dynamics we have prepared atomistic models of very thin layers of a-Si:H. We first study the onedimensional quantum confinement of a-Si:H slabs in vacuum, which is representative of a strong confinement regime. The Tauc band gap of a 16- $\AA$-thick layer is only slightly higher $(1.8 \mathrm{eV})$ than the bulk value $(1.7 \mathrm{eV})$. This points to the fact that the confinement effects are much weaker in an amorphous semiconductor than in a crystal. In the case of an 8 - $\AA$-thick layer the effects are more sizable $(2.1 \mathrm{eV})$.

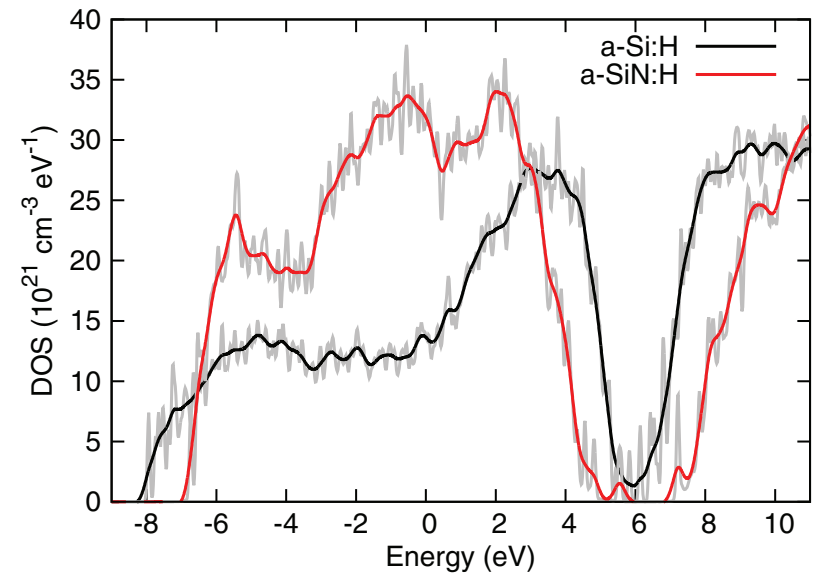

FIG. 7. (Color online) DOS of bulk silicon and nitride in the QWs model. The colored curves result from smoothing the calculated DOS (gray lines) with splines. The curves are calculated from site-projected DOS summed in slabs $8 \AA$ wide. 


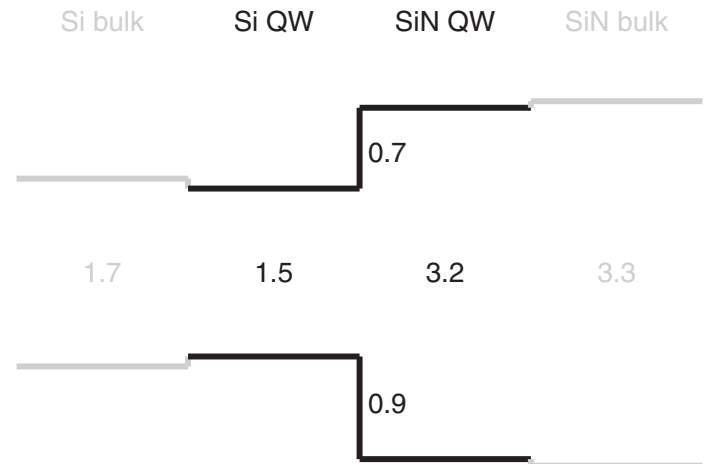

FIG. 8. Band diagram of the a-Si:H/a-SiN:H quantum well (black color). Band gaps of a-Si:H and a-SiN:H determined from bulk cells (gray color). All values are in electron volts. Note that adding the offset values to the a-Si:H band gap does not give the band gap of a-SiN:H precisely. This is due to round-off errors.

We have also prepared a model of QWs based on amorphous silicon and silicon nitride. Here the depth of the well depends on the band offsets between the two materials. This case is representative of the weak confinement regime. As expected we do not observe an increase in the band gap of a-Si:H. The gap even slightly decreases from 1.7 (bulk) to $1.5 \mathrm{eV}$. The decrease is attributed to the changes in structure due to an annealing cycle. The Tauc band-gap value is in apparent disagreement with optical measurement on an identical system. An increase in the experimental band gap can probably be explained as due to an increase of average nitrogen concentration.

The QWs model allows us to calculate band offsets between the two materials. The standard method used to extract offsets, for crystalline interfaces, could not be applied in this particular case. The reasons are twofold. The offsets cannot be calculated as rigid shifts of the bulk band structure due to annealing, which affects the structure. Secondly, the variation in the average potential is substantial and would introduce additional errors to the offsets. To circumvent the above-mentioned difficulties, we calculate the offsets from the site-projected DOS. We obtained 0.9 and $0.7 \mathrm{eV}$ for the valence and conduction band offsets, respectively. This means that the electrons and holes will encounter similar barriers during transport through the QWs structure.

\section{ACKNOWLEDGMENTS}

This work was carried out with a subsidy of the Dutch Ministry of Economic Affairs under EOS-LT program (Project No. EOSLT02028). We also acknowledge the support of the Foundation for Fundamental Research on Matter (FOM). Supercomputer facilities were provided by the National Computing Facilities Foundation (NCF). Both FOM and NCF receive financial support from the Netherlands Organization for Scientific Research (NWO).
[1] L. Eldada, Rev. Sci. Instrum. 75, 575 (2004).

[2] B. Abeles and T. Tiedje, Phys. Rev. Lett. 51, 2003 (1983).

[3] E. A. G. Hamers, M. N. van den Donker, B. Stannowski, R. Schlatmann, and G. J. Jongerden, Plasma Process. Polym. 4, 275 (2007).

[4] N. Ibaraki and H. Fritzsche, Phys. Rev. B 30, 5791 (1984).

[5] M. Hirose and S. Miyazaki, J. Non-Cryst. Solids 97, 23 (1987).

[6] S. Kalem, Phys. Rev. B 37, 8837 (1988).

[7] S. Miyazaki and M. Hirose, Philos. Mag. B 60, 23 (1989).

[8] H. Deki, S. Miyazaki, M. Ohmura, and M. Hirose, J. Non-Cryst. Solids 164, 841 (1993).

[9] S. Miyazaki, Y. Ihara, and M. Hirose, Phys. Rev. Lett. 59, 125 (1987).

[10] D. J. Lockwood, Z. H. Lu, and J. M. Baribeau, Phys. Rev. Lett. 76, 539 (1996).

[11] S. Nihonyanagi, K. Nishimoto, and Y. Kanemitsu, J. Non-Cryst. Solids 299, 1095 (2002).

[12] R. W. Collins and C. Y. Huang, Phys. Rev. B 34, 2910 (1986).

[13] M. Beaudoin, M. Meunier, and C. J. Arsenault, Phys. Rev. B 47, 2197 (1993).

[14] C. J. Arsenault, M. Meunier, M. Beaudoin, and B. Movaghar, Phys. Rev. B 44, 11521 (1991).

[15] N. Bernhard, B. Frank, B. Movaghar, and G. H. Bauer, Philos. Mag. B 70, 1139 (1994).

[16] N. Bernhard and G. H. Bauer, Phys. Rev. B 52, 8829 (1995).

[17] S. A. Koehler, Philos. Mag. B 77, 27 (1998).

[18] N. Kuzuu, K. Nagai, M. Tanaka, and Y. Tamai, Jpn. J. Appl. Phys. 44, 7539 (2005).
[19] E. J. Albenze and P. Clancy, Mol. Simul. 31, 11 (2005).

[20] N. Bernstein, M. J. Aziz, and E. Kaxiras, Phys. Rev. B 58, 4579 (1998).

[21] M. J. Caturla, T. Diaz De La Rubia, and G. H. Gilmer, J. Appl. Phys. 77, 3121 (1995).

[22] S. Izumi, S. Hara, T. Kumagai, and S. Sakai, Comput. Mater. Sci. 31, 279 (2004).

[23] T. Motooka, Nucl. Instrum. Methods Phys. Res., Sect. B 127, 244 (1997).

[24] M. Peressi, L. Colombo, and S. de Gironcoli, Phys. Rev. B 64, 193303 (2001)

[25] C. R. S. da Silva and A. Fazzio, Phys. Rev. B 64, 075301 (2001).

[26] B. Weber, D. M. Stock, and K. Gartner, Mater. Sci. Eng. B 71, 213 (2000)

[27] C. G. Van de Walle and L. H. Yang, J. Vac. Sci. Technol. B 13, 1635 (1995).

[28] A. Alkauskas and A. Pasquarello, Phys. B 401, 546 (2007).

[29] A. A. Demkov and O. F. Sankey, Phys. Rev. Lett. 83, 2038 (1999).

[30] F. Djurabekova and K. Nordlund, Phys. Rev. B 77, 115325 (2008).

[31] F. Giustino, Ph.D. thesis, École Polytechnique Fédérale de Lausanne, 2005.

[32] A. Pasquarello, M. Hybertsen, and R. Car, Nature (London) 396, 58 (1998).

[33] B. R. Tuttle, Phys. Rev. B 70, 125322 (2004).

[34] P. Broqvist, A. Alkauskas, and A. Pasquarello, Appl. Phys. Lett. 92, 132911 (2008) 
[35] M. Tosolini, L. Colombo, and M. Peressi, Phys. Rev. B 69, 075301 (2004).

[36] R. Car and M. Parrinello, Phys. Rev. Lett. 55, 2471 (1985).

[37] G. Kresse and J. Hafner, Phys. Rev. B 47, 558 (1993).

[38] G. Kresse and J. Furthmüller, Phys. Rev. B 54, 11169 (1996).

[39] P. E. Blöchl, Phys. Rev. B 50, 17953 (1994).

[40] G. Kresse and D. Joubert, Phys. Rev. B 59, 1758 (1999).

[41] J. P. Perdew, J. A. Chevary, S. H. Vosko, K. A. Jackson, M. R. Pederson, D. J. Singh, and C. Fiolhais, Phys. Rev. B 46, 6671 (1992).

[42] J. Heyd, J. E. Peralta, G. E. Scuseria, and R. L. Martin, J. Chem. Phys. 123, 174101 (2005).

[43] J. Heyd, G. E. Scuseria, and M. Ernzerhof, J. Chem. Phys. 118, 8207 (2003).

[44] J. Heyd, G. E. Scuseria, and M. Ernzerhof, J. Chem. Phys. 124, 219906 (2006).

[45] A. Baldereschi, Phys. Rev. B 7, 5212 (1973).

[46] M. Marsman, J. Paier, A. Stroppa, and G. Kresse, J. Phys.: Condens. Matter 20, 064201 (2008).

[47] J. E. Ortega and F. J. Himpsel, Phys. Rev. B 47, 2130 (1993).

[48] M. Welkowsky and R. Braunstein, Phys. Rev. B 5, 497 (1972).

[49] G. Onida, L. Reining, and A. Rubio, Rev. Mod. Phys. 74, 601 (2002).

[50] M. Shishkin and G. Kresse, Phys. Rev. B 75, 235102 (2007).
[51] K. Jarolimek, R. de Groot, G. A. de Wijs, and M. Zeman, Mater. Res. Soc. Symp. Proc. 1153, A04 (2009).

[52] K. Jarolimek, R. A. de Groot, G. A. de Wijs, and M. Zeman, Phys. Rev. B 82, 205201 (2010).

[53] F. Giorgis, C. F. Pirri, and E. Tresso, Thin Solid Films 307, 298 (1997).

[54] The interface energy is calculated by subtracting the total energies of isolated cells after rescaling (bulk material) from the total energy of the QWs model. The result is then divided by twice the interface area.

[55] R. A. Street, Hydrogenated Amorphous Silicon (Cambridge University Press, Cambridge, 1991).

[56] S. K. O’Leary, J. Mater. Sci.: Mater. Electron. 15, 401 (2004).

[57] M. Zeman, O. Isabella, F. D. Tichelaar, and S. L. Luxembourg, Phys. Status Solidi C 7, 1057 (2010).

[58] We performed tests on a single conventional unit cell of c-Si with one, two, three, or four cells of vacuum. The difference in band gap between the supercells with one and four cells of vacuum was, however, only $6 \mathrm{meV}$.

[59] A. Baldereschi, S. Baroni, and R. Resta, Phys. Rev. Lett. 61, 734 (1988).

[60] M. Peressi, A. Baldereschi, and S. Baroni, Characterization of Semiconductor Heterostructures and Nanostructures (Elsevier, New York, 2008), Chap. 2, p. 17. 